\title{
Canadian Revue
}

Journal of canadienne de
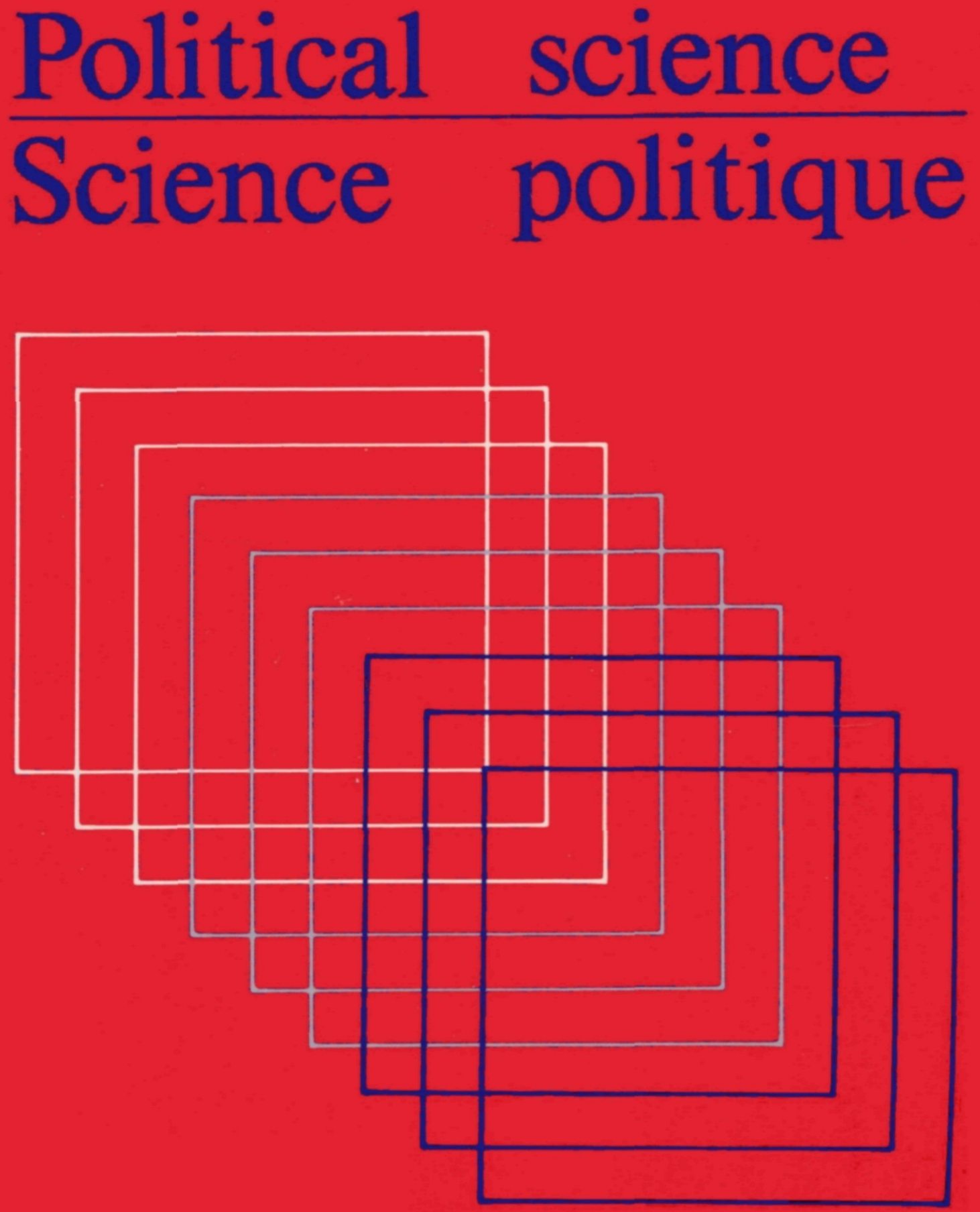

December 1984 décembre XVIIIshed online by Cambridge Univers 4 


\section{Canadian Journal of Political Science / Revue canadienne de science politique}

\section{EDITORIAL BOARD / COMITÉ DE RÉDACTION}

Robert Boardman (Dalhousie), co-editor

Grace Skogstad (St. Francis Xavier), review editor

Herman Bakvis (Dalhousie), assistant editor
Jean Crête (Laval), codirecteur

Graciela Ducatenzeiler (Montréal), secrétaire à la rédaction et responsable des recensions

John McMenemy, managing editor/directeur de la gestion, Wilfrid Laurier University

ADVISORY BOARD / CONSEIL CONSULTATIF

André Bernard (Université du Québec

Vincent Lemieux (Laval)

à Montréal)

Richard Johnston (British Columbia)

William Mathie (Brock)

Bahgat Korany (Montréal)

Janice Stein (Toronto)

Reginald Whitaker (Carleton)

Editorial correspondence in English should be directed to Professor Robert Boardman, Department of Political Science, Dalhousie University, Halifax, Nova Scotia B3H 4H6. Authors are requested to forward three copies of the manuscript together with three copies of an abstract of 100 words suitable for translation. All material must be double-spaced. Footnotes must be double-spaced and placed in a single listing at the end of the paper. Tables and diagrams must be submitted on separate pages. The manuscript will not be returned.

On doit adresser toute communication en français au sujet de la rédaction au Professeur Jean Crête, Département de science politique, Université Laval, Sainte-Foy, P.Q. GIK 7P4. Les auteurs envoient leur manuscrit, en trois exemplaires, accompagné d'un résumé, en trois exemplaires, ne dépassant pas 100 mots. Le tout doit être dactylographié à double interligne. Il faut que les notes apparaissent à double interligne, à la fin du texte, en ordre continu et sur des feuilles distinctes. Les tableaux et graphiques doivent être faits sur des pages séparées. Le manuscrit ne sera pas renvoyé.

Correspondence related to subscriptions should be directed to: Wilfrid Laurier University Press, Wilfrid Laurier University, Waterloo, Ontario N2L 3C5. All other business correspondence should be sent to The Managing Editor, c/o The Canadian Political Science Association/ACSP, 12 Henderson Avenue, University of Ottawa, Ottawa, Ontario KIN 6N5. / Les communications concernant les abonnements doivent être adressées à : Wilfrid Laurier University Press, Wilfrid Laurier University, Waterloo, Ontario N2L 3C5. Prière d'adresser toute autre communication de caractère commercial au Directeur de la gestion, a/s Association canadienne de Science politique/CPSA, 12, avenue Henderson, Université d'Ottawa, Ottawa, Ontario K1N 6N5.

The Journal acknowledges with gratitude the generous assistance of the Social Sciences and Humanities Research Council of Canada. / La Revue tient à remercier le Conseil de recherches en sciences humaines du Canada pour la généreuse subvention qu'il a bien voulu lui accorder.

INDEXED IN / INDEXÉE AU: International Political Science Abstracts / Documentation Politique Internationale; ABC POL SCI (Advance Bibliography of Contents: Political Science and Government); Périodex: index analytique de périodiques de langue française; Sociological Abstracts; United States Political Science Documents; Arts \& Humanities Citation Index (A\&HCI); Current Contents/Arts \& Humanities; PAIS Bulletin and PAIS Foreign Language Index; Index to Canadian Legal Periodical Literature (partial/partiel); Indian Book Review Digest (partial/partiel); International Political Science Information Service; IBZ (Internationale Bibliographie der Zeitschriftenliteratur); IBR (Internationale Bibliographie der Rezensionen).

The headquarters of the Canadian Political Science Association is at the University of Ottawa, Ottawa, Canada K1N 6N5. / Le siège sociale de l'Association canadienne de science politique est à l'Université d'Ottawa, Ottawa, Canada K1N 6N5. Le siège social de la Société québécoise de science politique est à / The headquarters of the Société québécoise de science politique is at the Université du Québec à Montréal, Montréal, Canada H3C 3P8.

(c) Canadian Political Science Association (l'Association canadienne de science politique) and/et la Société québécoise de science politique 1984

Published by / Publiée par Wilfrid Laurier University Press for/pour the Canadian Political Science Association (1'Association canadienne de science politique) and/et la Société québécoise de science politique

CN ISSN 0008-4239

Second class mail registration / Courrier recommandé de deuxième classe \#4011 


\section{Contents / Sommaire}

Women and the Welfare State

CAROLINE ANDREW 667

Minority Government in Ontario, 1975-1981: An Assessment

VAUGHAN LYON 685

On Retrieving Macpherson's Liberalism

JOHN W. SEAMAN and THOMAS J. LEWIS 707

Rousseau and the Domestication of Virtue

PETER EMBERLEY 731

Field Analyses / Orientations de la science politique

Les femmes dans les structures urbaines-aperçu d'un nouveau champ de recherche

DOMINIQUE MASSON 755

The Concept of Province-Building: A Critique

R. A. YOUNG, PHILIPPE FAUCHER, and ANDRÉ BLAIS 783

Recensions / Reviews 819

Notices / Avis 871

Index 877 


\section{Canadian Political Science Association / Association canadienne de science politique}

President/Président, Kal Holsti, University of British Columbia

Past President/Présidente sortante Caroline Andrew, University of Ottawa

President Elect/Président élu Frederick C. Engelmann, University of Alberta

Secretary-Treasurer/Secrétaire-trésorière Sharon Sutherland, Carleton University (on sabbatical)

CPSA/ACSP Secretariat

12 Henderson Avenue

University of Ottawa

Ottawa, Ontario KIN 6N5

Representative of Members-at-Large/

Représentant des conseillers

Pierre Fournier, Université du

Québec à Montréa!

Members-at-large/Conseillers 2-year term/mandat de deux ans: Barry Cooper (Calgary), Martha Fletcher (Government of Ontario), Jean-Pierre Gaboury (Ottawa), Alex McLeod (UQAM), Harald von Riekhoff (Carleton)

Société québécoise de science politique

Président, Edouard Cloutier Université de Montréal

Vice-président, Paul Noble McGill University

Trésorière, Diane Éthier Université d'Ottawa

Secrétaire Maurice Couture Université du Québec à Montréal C.P. 8888, Montréal, P.Q. H3C 3P8

Autres conseillers Gordon Mace (Laval), Jean Bernatchez (Laval), Benoit Godin (Laval), Clinton Archibald (Ottawa), Antoine Ambroise (Laval), Sylvie Paquerot (UQAM), Louise Quesnel (Laval) l-year term/mandat d' $u n$ an: William Chandler (McMaster), Pierre Fournier (UQAM), Linda Freeman (Carleton), Kenneth McRoberts (York), Donald Munton (CIIA)

Nominating Committee 1985 Comité de nomination

Maureen Covell (SFU), Dieter Hoehne

(Memorial), Evelyne Tardy (UQAM)

Invited guests/Invités

Robert Boardman (Dalhousie), co-editor of the Journal

Jean Crête (Laval), codirecteur de la Revue

André Bernard (UQAM), Chairperson of the Programme Committee/Président du Comité d'organisation du programme (1985)

Délégué de la Société québécoise de science politique

Joan Pond, Administrator/Administratrice

Invités

Jean Crête (Laval), Robert Boardman (Dalhousie), codirecteurs de la Revue Denis Monière, directeur de la revue Politique

Délégué de l'Association canadienne de science politique

Colette Désilets, secrétaire exécutive, Université du Québec à Montréal 